\title{
Strongly Correlated Fractional Quantum Hall Line Junctions
}

\author{
U. Zülicke ${ }^{1}$ and E. Shimshoni ${ }^{2,3}$ \\ ${ }^{1}$ Institut für Theoretische Festkörperphysik, Universität Karlsruhe, D-76128 Karlsruhe, Germany \\ ${ }^{2}$ Department of Mathematics-Physics, University of Haifa at Oranim, Tivon 36006, Israel \\ ${ }^{3}$ Department of Physics and Astronomy, Rutgers University, Piscataway, NJ 08854-8019
}

(Dated: October 24, 2018)

\begin{abstract}
We have studied a clean finite-length line junction between interacting counterpropagating singlebranch fractional-quantum-Hall edge channels. Exact solutions for low-lying excitations and transport properties are obtained when the two edges belong to quantum Hall systems with different filling factors and interact via the long-range Coulomb interaction. Charging effects due to the coupling to external edge-channel leads are fully taken into account. Conductances and power laws in the current-voltage characteristics of tunneling are strongly affected by inter-edge correlations.
\end{abstract}

Two-dimensional (2D) electron systems exhibit incompressibilities when their sheet density $n_{0}$ is commensurate with the value $B$ of perpendicular magnetic field such that the filling factor $\nu=2 \pi \hbar n_{0} /|e B|$ is integer or equal to certain fractions [1, 2]. Such an incompressible phase is characterized by a quantized value of the Hall resistance and low-lying excitations that are localized at the boundary of the 2D system [3, 4, 5, 6, 7]. When $\nu$ is the inverse of an odd integer, these quantum-Hall $(\mathrm{QH})$ edge excitations have been shown [8] to be isomorphous to those of a single-branch chiral one-dimensional (1D) electron system 9]. Similar to their nonchiral counterparts that are realized, e.g., in semiconductor quantum wires 10] or carbon nanotubes [11], QH edges are expected [8] to exhibit power laws in electronic correlation functions that are the hallmark of Luttinger-liquid behavior [12]. Virtually no other quasi-1D electron system, however, matches the versatility of $\mathrm{QH}$ edges in tailoring their electronic properties which can be achieved, e.g., simply by adjusting the magnetic field and/or appropriate nanostructuring techniques. Recent applications of the cleaved-edge overgrowth method [13, 14] have succeeded in creating extended uniform tunnel junctions between two integer $\mathrm{QH}$ edges 15 or a $2 \mathrm{D}$ electron system and a QH edge [16]. Besides opening up new possibilities for electron tunneling spectroscopy [17], these new sample geometries enable the controlled experimental study of the interplay between tunneling and interaction effects in low dimensions [18, 19, 20, 21] that cannot be performed in conventional systems.

A diverse set of electron-correlation effects has been proposed for $\mathrm{QH}$ line junctions where both edge channels belong to $\mathrm{QH}$ systems having the same filling factor 22, 23, 24, 25, 26]. In our work presented here, we consider the case of a clean junction where the filling factors $\nu_{\mathrm{R}}$ and $\nu_{\mathrm{L}}$, characterizing the respective rightmoving and left-moving edges, are different. Such a situation could be realized, e.g., in samples with two mutually perpendicular $2 \mathrm{D}$ electron systems 16 and a magnetic field properly adjusted in magnitude and direction. In the experimentally realistic limit where electrons from the two edges interact strongly via Coulomb interactions, low-lying excitations are represented by two decoupled bosonic modes that have opposite chirality. One of them, the charged mode, corresponds to fluctuations in the total electron density in the two edges and is free. The dynamics of its orthogonal complement, the neutral mode, turns out to be governed by realizations of chiral sineGordon models [27, 28] that can be solved exactly for certain values of the parameter $\tilde{\nu}=\nu_{\mathrm{R}} \nu_{\mathrm{L}} /\left|\nu_{\mathrm{R}}-\nu_{\mathrm{L}}\right|$. We use these exact solutions to calculate transport through the line junction and find that the strong inter-edge correlations significantly affect the conductance and tunneling resonances arising from translational invariance along the barrier.

Starting point of our calculations is the Hamiltonian for our model of the line junction. (See Fig. 1) It reads $H_{\mathrm{J}}=\int_{-L / 2}^{L / 2} d x\left(\mathcal{H}_{\mathrm{R}}+\mathcal{H}_{\mathrm{L}}+\mathcal{H}_{\text {int }}+\mathcal{H}_{\text {tun }}\right)$ where $\mathcal{H}_{\mathrm{R} / \mathrm{L}}$ describe isolated edge channels, $\mathcal{H}_{\text {int }}$ the inter-edge interaction, and $\mathcal{H}_{\text {tun }}$ uniform tunneling across the barrier:

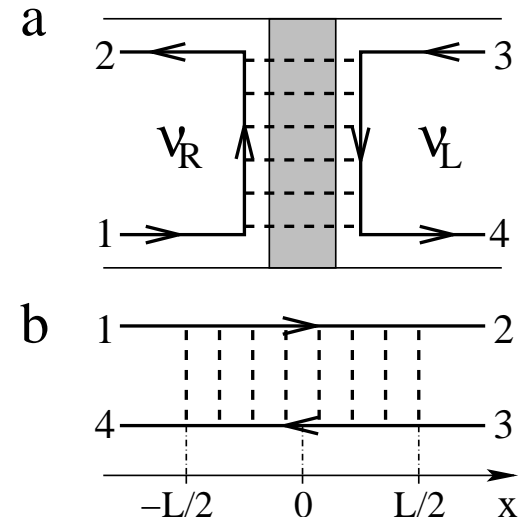

FIG. 1: Schematic layout of a quantum-Hall line junction. An extended uniform tunnel barrier couples counterpropagating single-branch edge channels from two $2 \mathrm{D}$ electron systems that have different fractional filling factors $\nu_{\mathrm{R}}$ and $\nu_{\mathrm{L}}$. (See panel a.) We model this situation by two parallel infinite chiral fractional edge channels that are coupled via uniform tunneling and Coulomb interactions along the finite junction region where $|x| \leq L / 2$. Outside the junction, edge branches correspond to leads connecting to external reservoirs. 


$$
\begin{aligned}
\mathcal{H}_{\mathrm{R} / \mathrm{L}} & =\frac{1}{4 \pi}\left\{\hbar v_{\mathrm{R} / \mathrm{L}}^{(0)}+\frac{\nu_{\mathrm{R} / \mathrm{L}}}{2 \pi} U\right\}\left[\partial_{x} \phi_{\mathrm{R} / \mathrm{L}}\right]^{2}, \\
\mathcal{H}_{\text {int }} & =\frac{\sqrt{\nu_{\mathrm{R}} \nu_{\mathrm{L}}}}{4 \pi^{2}} \lambda U \partial_{x} \phi_{\mathrm{R}} \partial_{x} \phi_{\mathrm{L}} \\
\mathcal{H}_{\text {tun }} & =t\left\{\psi_{\mathrm{R}}^{\dagger}(x) \psi_{\mathrm{L}}(x)+\text { H.c. }\right\}
\end{aligned}
$$

Here we denote the second-quantized annihilation operators for electrons from the two edges as $\psi_{\mathrm{R} / \mathrm{L}}$. The chiral bosonic phase fields $\phi_{\mathrm{R} / \mathrm{L}}(x)$ are related to the respective edge densities via $\varrho_{\mathrm{R} / \mathrm{L}}=\psi_{\mathrm{R} / \mathrm{L}}^{\dagger} \psi_{\mathrm{R} / \mathrm{L}}=$ $\sqrt{\nu_{\mathrm{R} / \mathrm{L}}} \partial_{x} \phi_{\mathrm{R} / \mathrm{L}} /(2 \pi)$ and obey the commutation relations $\left[\phi_{\mathrm{R} / \mathrm{L}}(x), \phi_{\mathrm{R} / \mathrm{L}}\left(x^{\prime}\right)\right]= \pm i \pi \operatorname{sgn}\left(x-x^{\prime}\right)$. The bare (confinement-induced) electron velocities in the two edge channels are given by $v_{\mathrm{R} / \mathrm{L}}^{(0)}, U$ is the matrix element of screened Coulomb interactions, and we have taken into account the generally different interaction strengths within and between the edges by a factor $0<\lambda \leq$ 1. It is straightforward to diagonalize the part $H_{\mathrm{J}}-$ $\int_{-L / 2}^{L / 2} d x \mathcal{H}_{\text {tun }}$, and using the bosonization identity [29]

$$
\psi_{\mathrm{R} / \mathrm{L}}(x)=\sqrt{z_{\mathrm{R} / \mathrm{L}}} \mathcal{F}_{\mathrm{R} / \mathrm{L}} e^{i x \frac{Y_{\mathrm{R} / \mathrm{L}}}{\ell^{2}} \pm i \frac{\phi_{\mathrm{R} / \mathrm{L}}(x)}{\left(\nu_{\mathrm{R} / \mathrm{L}}\right)^{1 / 2}}},
$$

the tunneling term $\mathcal{H}_{\text {tun }}$ can be expressed solely in terms of bosonic phase fields as well. [In Eq. (2), $z_{\mathrm{R} / \mathrm{L}}$ are normalization constants, $\mathcal{F}_{\mathrm{R} / \mathrm{L}}$ Klein factors, $Y_{\mathrm{R} / \mathrm{L}}$ the guiding-center coordinates of edge electrons in the direction perpendicular to the junction, and $\ell=\sqrt{\hbar c /|e B|}$ the magnetic length.] However, explicit expressions for normal modes in the most general case are unilluminating. We therefore proceed immediately to the special case of interest to us where strong Coulomb interactions dominate the bare electron velocities in each branch, i.e., $\hbar v_{\mathrm{R} / \mathrm{L}}^{(0)} \ll U$, and the distance between the two edges is of the order of or smaller than $\ell$ such that $\lambda \rightarrow 1$. It is then possible to rewrite the $\mathrm{QH}-$ junction Hamiltonian as $H_{\mathrm{J}}=\int_{-L / 2}^{L / 2} d x\left(\mathcal{H}_{\mathrm{c}}+\mathcal{H}_{\mathrm{n}}\right)$ with the contributions

$$
\begin{aligned}
\mathcal{H}_{\mathrm{c}} & =\frac{\hbar v_{\mathrm{c}}}{4 \pi}\left[\partial_{x} \phi_{\mathrm{c}}\right]^{2} \\
\mathcal{H}_{\mathrm{n}} & =\frac{\hbar v_{\mathrm{n}}}{4 \pi}\left[\partial_{x} \phi_{\mathrm{n}}\right]^{2}+2 t \sqrt{z_{\mathrm{R}} z_{\mathrm{L}}} \cos \left(\frac{\phi_{\mathrm{n}}}{\sqrt{\tilde{\nu}}}+\frac{x \Delta}{\ell^{2}}\right)
\end{aligned}
$$

for independent $\mathrm{c}$ (harged) and $\mathrm{n}$ (eutral) modes [36]. Their corresponding phase fields obey commutation relations $\left[\phi_{\mathrm{c} / \mathrm{n}}(x), \phi_{\mathrm{c} / \mathrm{n}}\left(x^{\prime}\right)\right]= \pm \operatorname{sgn}\left(\nu_{\mathrm{R}}-\nu_{\mathrm{L}}\right) i \pi \operatorname{sgn}\left(x-x^{\prime}\right)$ and are related to the densities of the original rightmoving and left-moving edge electrons via

$$
\varrho_{\mathrm{R} / \mathrm{L}}=\frac{ \pm \operatorname{sgn}\left(\nu_{\mathrm{R}}-\nu_{\mathrm{L}}\right)}{2 \pi \sqrt{\left|\nu_{\mathrm{R}}-\nu_{\mathrm{L}}\right|}}\left(\nu_{\mathrm{R} / \mathrm{L}} \partial_{x} \phi_{\mathrm{c}}-\sqrt{\nu_{\mathrm{R}} \nu_{\mathrm{L}}} \partial_{x} \phi_{\mathrm{n}}\right) .
$$

The velocities are $v_{\mathrm{c}}=\left|\nu_{\mathrm{R}}-\nu_{\mathrm{L}}\right| U /(2 \pi \hbar)$ and $v_{\mathrm{n}}=$ $\left(\nu_{\mathrm{R}} v_{\mathrm{L}}^{(0)}+\nu_{\mathrm{L}} v_{\mathrm{R}}^{(0)}\right) /\left|\nu_{\mathrm{R}}-\nu_{\mathrm{L}}\right|$, and we used the abbreviation
$\Delta:=Y_{\mathrm{R}}-Y_{\mathrm{L}}$. We see from Eqs. (3) that the charged mode is free while the neutral-mode dynamics is that of a chiral sine-Gordon model [27, 28]. Before obtaining its solution, we interject an elaboration on observables related to electron transport.

To discuss transport properties, it is necessary to consider continuity equations and chemical potentials for the various edge densities. Denoting time by $\tau$, the operator for particle current (per unit length) across the barrier is

$$
\begin{aligned}
\mathcal{I}_{\mathrm{J}}(x) & =-\frac{d}{d \tau} \varrho_{\mathrm{R}}(x)=\frac{d}{d \tau} \varrho_{\mathrm{L}}(x), \\
& =i \frac{t}{\hbar}\left\{\psi_{\mathrm{R}}^{\dagger}(x) \psi_{\mathrm{L}}(x)-\text { H.c. }\right\},
\end{aligned}
$$

In our limit of interest, its bosonized expression depends on the neutral mode only,

$$
\mathcal{I}_{\mathrm{J}}(x)=2 \frac{t}{\hbar} \sqrt{z_{\mathrm{R}} z_{\mathrm{L}}} \sin \left(\frac{\phi_{\mathrm{n}}}{\sqrt{\tilde{\nu}}}+\frac{x \Delta}{\ell^{2}}\right) .
$$

It is straightforward to find a continuity equation for density $\varrho_{\mathrm{c}}:=\partial_{x} \phi_{\mathrm{c}} /(2 \pi)$ associated with the charged mode,

$$
\begin{aligned}
0 & =\frac{d}{d \tau} \frac{\varrho_{\mathrm{R}}+\varrho_{\mathrm{L}}}{\sqrt{\left|\nu_{\mathrm{R}}-\nu_{\mathrm{L}}\right|}} \equiv \frac{d}{d \tau} \varrho_{\mathrm{c}}, \\
& =\left\{\partial_{\tau}+\operatorname{sgn}\left(\nu_{\mathrm{R}}-\nu_{\mathrm{L}}\right) v_{\mathrm{c}} \partial_{x}\right\} \varrho_{\mathrm{c}},
\end{aligned}
$$

and the corresponding one for $\varrho_{\mathrm{n}}:=\partial_{x} \phi_{\mathrm{n}} /(2 \pi)$,

$$
\begin{aligned}
0 & =\frac{d}{d \tau} \varrho_{\mathrm{n}}-\frac{\operatorname{sgn}\left(\nu_{\mathrm{R}}-\nu_{\mathrm{L}}\right)}{\sqrt{\tilde{\nu}}} \mathcal{I}_{\mathrm{J}}, \\
& =\left\{\partial_{\tau}-\operatorname{sgn}\left(\nu_{\mathrm{R}}-\nu_{\mathrm{L}}\right) v_{\mathrm{n}} \partial_{x}\right\} \varrho_{\mathrm{n}} .
\end{aligned}
$$

In the stationary regime, densities have no explicit time dependence. The above continuity equations then imply that they assume constant values $\bar{\varrho}_{\mathrm{c} / \mathrm{n}}$ along the junction. We can also derive expressions for local chemical potentials $\mu_{\mathrm{c} / \mathrm{n}}:=\delta H_{\mathrm{J}} / \delta \varrho_{\mathrm{c} / \mathrm{n}} \equiv \mp i \operatorname{sgn}\left(\nu_{\mathrm{R}}-\nu_{\mathrm{L}}\right)\left[H_{\mathrm{J}}, \phi_{\mathrm{c} / \mathrm{n}}\right]$ associated with the charged and neutral modes:

$$
\begin{aligned}
\mu_{\mathrm{c}}(x)= & 2 \pi \hbar v_{\mathrm{c}} \varrho_{\mathrm{c}}(x), \\
\mu_{\mathrm{n}}(x)= & 2 \pi \hbar v_{\mathrm{n}} \varrho_{\mathrm{n}}(x) \\
& +\frac{\pi \hbar}{\sqrt{\tilde{\nu}}} \int_{-\frac{L}{2}}^{\frac{L}{2}} d x^{\prime} \mathcal{I}_{\mathrm{J}}\left(x^{\prime}\right) \operatorname{sgn}\left(x-x^{\prime}\right) .
\end{aligned}
$$

Specializing these to the stationary limit, we find

$$
\begin{aligned}
\mu_{\mathrm{c}}(x) & =2 \pi \hbar v_{\mathrm{c}} \bar{\varrho}_{\mathrm{c}}=\text { const. } \\
\mu_{\mathrm{n}}( \pm L / 2) & =2 \pi \hbar v_{\mathrm{n}} \bar{\varrho}_{\mathrm{n}} \pm \frac{\pi \hbar}{\sqrt{\tilde{\nu}}} I_{\mathrm{J}}
\end{aligned}
$$

where $I_{\mathrm{J}}:=\int_{-L / 2}^{L / 2} d x \mathcal{I}_{\mathrm{J}}(x)$ is the total current flowing through the junction. The values of $\bar{\varrho}_{\mathrm{c} / \mathrm{n}}$ and $I_{\mathrm{J}}$ can be related to chemical potentials in the external leads that 
connect the QH junction to reservoirs (see Fig. 1), which we proceed to show now.

In a real setup as sketched in Fig. 10, edge channels away from the junction are not coupled via tunneling or interactions anymore. However, for each of these chiral 1D leads, Coulomb interactions between electrons at the same edge still give the dominant contribution to the edge-magnetoplasmon velocity [30]. We therefore model the dynamics of electrons in regions $|x|>L / 2$ by the lead Hamiltonian $H_{\mathrm{E}}=\left\{\int_{-\infty}^{-L / 2}+\int_{L / 2}^{\infty}\right\} d x\left(\mathcal{H}_{\mathrm{R}}+\mathcal{H}_{\mathrm{L}}\right)$. While $H_{\mathrm{E}}$ is not diagonal in the charged and neutral modes, their corresponding chemical potentials are still well-defined in the lead regions. As the densities in each of the four chiral edge-channel leads cannot change, chemical potentials remain constant as well 31. Assuming again $U \gg \hbar v_{\mathrm{R} / \mathrm{L}}^{(0)}$, we find for these (in a compact notation where $\mu_{i / j}$ means $\mu_{i}$ or $\mu_{j}$, respectively)

$$
\begin{aligned}
& \left.\mu_{\mathrm{c}}\right|_{x \lesseqgtr \mp \frac{L}{2}}=\frac{\nu_{\mathrm{R}} \mu_{1 / 2}-\nu_{\mathrm{L}} \mu_{4 / 3}}{\sqrt{\left|\nu_{\mathrm{R}}-\nu_{\mathrm{L}}\right|}} \operatorname{sgn}\left(\nu_{\mathrm{R}}-\nu_{\mathrm{L}}\right),(11 \mathrm{a}) \\
& \left.\mu_{\mathrm{n}}\right|_{x>\mp \frac{L}{2}}=-\sqrt{\tilde{\nu}}\left(\mu_{1 / 2}-\mu_{4 / 3}\right) \operatorname{sgn}\left(\nu_{\mathrm{R}}-\nu_{\mathrm{L}}\right)(11 \mathrm{~b}
\end{aligned}
$$

Demanding continuity of $\mu_{\mathrm{c} / \mathrm{n}}$ at $x= \pm L / 2$ yields four linear relations involving the chemical potentials in the four leads, the constants $\bar{\varrho}_{\mathrm{c} / \mathrm{n}}$, and $I_{\mathrm{J}}$. After some straightforward algebra, we obtain the expressions

$$
\begin{aligned}
\mu_{2 / 4} & =\mu_{1 / 3} \pm \frac{2 \pi \hbar}{\nu_{\mathrm{R} / \mathrm{L}}} I_{\mathrm{J}} \\
v_{\mathrm{c}} \bar{\varrho}_{\mathrm{c}} & =\frac{\nu_{\mathrm{R}} \mu_{1}-\nu_{\mathrm{L}} \mu_{3}+2 \pi \hbar I_{\mathrm{J}}}{2 \pi \hbar \sqrt{\left|\nu_{\mathrm{R}}-\nu_{\mathrm{L}}\right|}} \operatorname{sgn}\left(\nu_{\mathrm{R}}-\nu_{\mathrm{L}}\right), \\
v_{\mathrm{n}} \bar{\varrho}_{\mathrm{n}} & =\frac{\mu_{1}-\mu_{3}+\left(\frac{\pi \hbar}{\nu_{\mathrm{R}}}+\frac{\pi \hbar}{\nu_{\mathrm{L}}}\right) I_{\mathrm{J}}}{-2 \pi \hbar / \sqrt{\tilde{\nu}}} \operatorname{sgn}\left(\nu_{\mathrm{R}}-\nu_{\mathrm{L}}\right)(
\end{aligned}
$$

relating the 'output' chemical potentials $\mu_{2 / 4}$ as well as the constants $\bar{\varrho}_{\mathrm{c} / \mathrm{n}}$ to the experimentally adjustable 'input' chemical potentials $\mu_{1 / 3}$ and the a priori unknown current $I_{\mathrm{J}}$ 37]. To obtain the full solution for the transport problem of our finite $\mathrm{QH}$ junction that is attached to edge-channel leads, we are now left with the task to determine $\bar{\varrho}_{\mathrm{n}}$ and $I_{\mathrm{J}}$ self-consistently using the dynamics of the neutral mode expressed in Eqs. (3b), (6), (9b), and (12c). We now show how this can be achieved.

The Hamiltonian of the neutral mode [given by Eq. [3b] is a realization of recently discussed [27, 28] chiral sine-Gordon models. In contrast to the well-known nonchiral sine-Gordon model 32], the quantum character of its dynamics arises because the bosonic field entering the cosine term does not commute with itself. Possible values of the parameter $\tilde{\nu}$ for the $\mathrm{QH}$ line junction discussed here can be parameterized by two non-negative integers $m, m^{\prime}$ such that $1 / \tilde{\nu}=2\left|m-m^{\prime}\right|$. These values correspond to $\mathrm{QH}$ junctions between systems having filling factors $1 /(2 m+1)$ and $1 /\left(2 m^{\prime}+1\right)$, respectively.
It has been shown [28] that the cosine term constitutes only an irrelevant perturbation to the dynamics of the chiral bosonic field when $\left|m-m^{\prime}\right|>2$. More interesting, and easier to realize experimentally, are the two cases $1 / \tilde{\nu} \in\{2,4\}$ where the cosine term turns out to be relevant or marginal, respectively, and exact solutions for its dynamics can be found [27, 28].

Lets first consider the case $\tilde{\nu}=1 / 2$ realized, e.g., at a line junction between $\mathrm{QH}$ systems having filling factors 1 and $1 / 3$, respectively. Introducing an auxiliary chiral boson field $\eta(x)$ that has the same chirality and velocity as $\phi_{\mathrm{n}}$, we can define fictitious chiral fermion operators $\Psi_{\uparrow / \downarrow}$ via an inverse bosonization identity $\Psi_{\sigma} \propto \exp \left\{-i \operatorname{sgn}\left(\nu_{\mathrm{R}}-\nu_{\mathrm{L}}\right)\left[\eta+\sigma \phi_{\mathrm{n}}\right] / \sqrt{2}\right\}$. Note that the pseudospin degree of freedom indexed by $\sigma$ is not related to the spin of the original electrons in the sample. In the new fictitious-fermion degrees of freedom, Eqs. (3b) and (6) are quadratic:

$$
\begin{aligned}
& \mathcal{H}_{\mathrm{n}}^{\prime}= \sum_{\sigma} \Psi_{\sigma}^{\dagger}\left[i \operatorname{sgn}\left(\nu_{\mathrm{R}}-\nu_{\mathrm{L}}\right) \hbar v_{\mathrm{n}} \partial_{x}\right] \Psi_{\sigma} \\
&+t\left(\Psi_{\uparrow}^{\dagger} \Psi_{\downarrow} e^{i \frac{x \Delta}{\ell^{2}} \operatorname{sgn}\left(\nu_{\mathrm{R}}-\nu_{\mathrm{L}}\right)}+\text { H.c. }\right) \\
& \mathcal{I}_{\mathrm{J}}^{\prime}=-i \frac{t}{\hbar}\left(\Psi_{\uparrow}^{\dagger} \Psi_{\downarrow} e^{i \frac{x \Delta}{\ell^{2}} \operatorname{sgn}\left(\nu_{\mathrm{R}}-\nu_{\mathrm{L}}\right)}-\text { H.c. }\right)
\end{aligned}
$$

In addition, density and chemical potential of the neutral mode are related to corresponding fictitious-fermion quantities via $\Psi_{\uparrow}^{\dagger} \Psi_{\uparrow}-\Psi_{\downarrow}^{\dagger} \Psi_{\downarrow} \equiv \sqrt{2} \varrho_{\mathrm{n}}$ and $\mu_{\uparrow}-\mu_{\downarrow} \equiv$ $\sqrt{2} \mu_{\mathrm{n}}$. Note that only the pseudospin sector of the new fermionic theory has any bearing for real observations, while the pseudocharge sector described by the auxiliary field $\eta$ is hidden. We see that the problem of the original line junction between two strongly interacting edge branches with opposite chirality has been mapped onto that of tunneling between two branches of noninteracting and chiral fermions. The latter is readily solved using standard methods. For example, when tunneling is weak enough such that it is possible to neglect the contribution $\propto I_{\mathrm{J}}$ in Eq. (12C), we find $e^{2} I_{\mathrm{J}}=G_{\mathrm{J}}\left(\mu_{1}-\mu_{3}\right)$ with the linear electric conductance

$$
G_{\mathrm{J}}=\frac{e^{2}}{2 \pi \hbar} \frac{\sin ^{2}\left[\frac{\pi L}{L_{t}} \sqrt{1+\xi^{2}}\right]}{1+\xi^{2}} .
$$

Here $L_{t}=\pi \hbar v_{\mathrm{n}} /|t|$ is a fundamental length scale set by tunneling, and $\xi=\hbar v_{\mathrm{n}} \Delta /\left(2|t| \ell^{2}\right)$ a resonance parameter: $G_{\mathrm{J}}$ is maximal for $\xi \rightarrow 0$ which corresponds to the resonance condition where both energy and 1D momentum are conserved in a tunneling event 38. The obtained linear dependence of $I_{\mathrm{J}}$ on $\mu_{1}-\mu_{3}$ has to be contrasted with the power law $I_{\mathrm{J}} \propto\left(\mu_{1}-\mu_{3}\right)^{\nu_{\mathrm{R}}^{-1}+\nu_{\mathrm{L}}^{-1}-1}$ that is to be expected for momentum-resolved tunneling at a line junction between noninteracting chiral-Luttingerliquid 8 edge channels. Furthermore, chirality of the effective tunneling problem described by Eqs. (13) results in charge oscillations along the junction similar to 
those predicted for QH bilayer systems [28] and parallel quantum wires 33 .

Before concluding, we discuss the other nontrivial case $\tilde{\nu}=1 / 4$ which is realized, e.g., when $\nu_{\mathrm{R}}=1$ and $\nu_{\mathrm{L}}=1 / 5$. Again it is possible to solve the problem by refermionization [28]. Defining $\psi_{\mathrm{n}}(x)=$ $\sqrt{z_{\mathrm{n}}} \mathcal{F}_{\mathrm{n}} \exp \left\{-i \operatorname{sgn}\left(\nu_{\mathrm{R}}-\nu_{\mathrm{L}}\right)\left[\phi_{\mathrm{n}}(x)+x \Delta / 2 \ell^{2}\right]\right\}$ and using the identity [28] $\psi_{\mathrm{n}}(x) i \partial_{x} \psi_{\mathrm{n}}(x)=2 \pi \operatorname{sgn}\left(\nu_{\mathrm{R}}-\right.$ $\left.\nu_{\mathrm{L}}\right) z_{\mathrm{n}}^{2} \mathcal{F}_{\mathrm{n}}^{2} \exp \left\{-\left[2 i \phi_{\mathrm{n}}(x)+i x \Delta / \ell^{2}\right]\right\}$, we can rewrite Eqs. (3b) and (6) as quadratic forms in the fictitious chiral Dirac fermion $\psi_{\mathrm{n}}$. It turns out to be useful to utilize its decomposition in terms of Majorana fermions, $\psi_{\mathrm{n}}=\left(\chi_{+}+i \chi_{-}\right) / \sqrt{2}$, which yields

$$
\begin{aligned}
& \mathcal{H}_{\mathrm{n}}^{\prime \prime}=\frac{1}{2} \sum_{r= \pm} \chi_{r}\left(i \hbar v_{r} \partial_{x}\right) \chi_{r}-\frac{\hbar v_{\mathrm{n}} \Delta}{\ell^{2}} i \chi_{+} \chi_{-}, \\
& \mathcal{I}_{\mathrm{J}}^{\prime \prime}=\frac{-i \tilde{t}}{2 \pi \hbar} \operatorname{sgn}\left(\nu_{\mathrm{R}}-\nu_{\mathrm{L}}\right)\left(\chi_{+} \partial_{x} \chi_{-}+\chi_{-} \partial_{x} \chi_{+}\right)
\end{aligned}
$$

where $v_{ \pm}=\operatorname{sgn}\left(\nu_{\mathrm{R}}-\nu_{\mathrm{L}}\right)\left[v_{\mathrm{n}} \pm \tilde{t} / \pi\right]$ with $\tilde{t}=t \sqrt{z_{\mathrm{R}} z_{\mathrm{L}}} / z_{\mathrm{n}}^{2}$. The Hamiltonian $\mathcal{H}_{\mathrm{n}}^{\prime \prime}$ is easily diagonalized, and the occupation-number distribution for Majorana fermions in reciprocal space is fixed by the requirement $i \chi_{+} \chi_{-}=$ $\bar{\rho}_{\mathrm{n}}$. The transport problem can then be solved exactly again. A complete analysis is left to a later publication 234; here we can only mention results for $\Delta=0$ and in the limit of weak tunneling where it is possible to neglect the contribution proportional to $I_{\mathrm{J}}$ in Eq. (12C). Quite different from the above considered case of $\tilde{\nu}=1 / 2$, we find here that $I_{\mathrm{J}}$ is oscillating in time with period $2(\pi \hbar)^{2} v_{n} /\left(\tilde{t}\left|\mu_{1}-\mu_{3}\right|\right)$. A similar dephasing effect as observed [35] in QH line junctions where $\nu_{\mathrm{R}}=\nu_{\mathrm{L}}=1$ leads to the temporal decay of the oscillation amplitude.

In summary, we have obtained exact solutions for transport through finite $\mathrm{QH}$ line junctions where the two edge channels belong to systems having different filling factors $\nu_{\mathrm{R}}$ and $\nu_{\mathrm{L}}$. Charging effects have been treated fully self-consistently by imposing appropriate boundary conditions for chemical potentials in the attached edge-channel leads. Strong coupling of edge channels via Coulomb interactions in a junction with $\tilde{\nu} \equiv \frac{\nu_{\mathrm{R}} \nu_{\mathrm{L}}}{\left|\nu_{\mathrm{R}}-\nu_{\mathrm{L}}\right|}=$ $1 / 2$ gives rise to a linear IV-characteristics for tunneling, as opposed to the power law expected in the absence of inter-edge correlations. At junctions with $\tilde{\nu}=1 / 4$, tunneling currents oscillate in time.

We thank N. Andrei, C. Chamon and V. Tripathi for useful discussions. This work was supported in part by the German Science Foundation (DFG) through Grant No. ZU 116/1 and the German Federal Ministry of Education and Research (BMBF) in the framework of the DIP program.

[1] R. E. Prange and S. M. Girvin, eds., The Quantum Hall Effect (Springer, New York, 1990), 2nd ed.
[2] S. Das Sarma and A. Pinczuk, eds., Perspectives in the Quantum Hall Effects (Wiley, New York, 1997).

[3] B. I. Halperin, Phys. Rev. B 25, 2185 (1982).

[4] A. H. MacDonald and P. Středa, Phys. Rev. B 29, 1616 (1984).

[5] A. H. MacDonald, Phys. Rev. Lett. 64, 220 (1990).

[6] X. G. Wen, Phys. Rev. B 41, 12838 (1990).

[7] J. Fröhlich and A. Zee, Nucl. Phys. B B364, 517 (1991).

[8] X. G. Wen, Int. J. Mod. Phys. B 6, 1711 (1992).

[9] J. Voit, Rep. Prog. Phys. 57, 977 (1994).

[10] A. Yacoby et al., Phys. Rev. Lett. 77, 4612 (1996).

[11] J. W. G. Wilder et al., Nature 391, 59 (1998).

[12] F. D. M. Haldane, J. Phys. C 14, 2585 (1981).

[13] L. Pfeiffer et al., Appl. Phys. Lett. 56, 1697 (1990).

[14] M. Grayson et al., Solid-State Electron. 40, 233 (1996).

[15] W. Kang et al., Nature 403, 59 (1999).

[16] M. Huber et al., Physica E 12, 125 (2001).

[17] U. Zülicke, E. Shimshoni, and M. Governale, Phys. Rev. B 65, 241315(R) (2002).

[18] A. M. Finkel'stein and A. I. Larkin, Phys. Rev. B 47, 10461 (1993).

[19] M. Fabrizio, Phys. Rev. B 48, 15838 (1993).

[20] D. G. Clarke, S. P. Strong, and P. W. Anderson, Phys. Rev. Lett. 72, 3218 (1994).

[21] H. J. Schulz, Phys. Rev. B 53, R2959 (1996).

[22] S. R. Renn and D. P. Arovas, Phys. Rev. B 51, 16832 (1995).

[23] C. L. Kane and M. P. A. Fisher, Phys. Rev. B 56, 15231 (1997)

[24] A. Mitra and S. M. Girvin, Phys. Rev. B 64, 041309(R) (2001).

[25] M. Kollar and S. Sachdev, Phys. Rev. B 65, 121304 (2002).

[26] E.-A. Kim and E. Fradkin, cond-mat/0205629.

[27] U. Zülicke, Phys. Rev. Lett. 83, 5330 (1999).

[28] J. D. Naud, L. P. Pryadko, and S. L. Sondhi, Nucl. Phys. B 565, 572 (2000).

[29] J. von Delft and H. Schoeller, Ann. Phys. (Leipzig) 7, 225 (1998).

[30] U. Zülicke and A. H. MacDonald, Phys. Rev. B 54, 16813 (1996).

[31] A. Yu. Alekseev, V. V. Cheianov, and J. Fröhlich, Phys. Rev. Lett. 81, 3503 (1998).

[32] A. M. Tsvelik, Quantum Field Theory in Condensed Matter Physics (Cambridge U. Press, Cambridge, UK, 1995).

[33] D. Boese, M. Governale, A. Rosch, and U. Zülicke, Phys. Rev. B 64, 085315 (2001).

[34] U. Zülicke and E. Shimshoni, in preparation.

[35] T.-L. Ho, Phys. Rev. B 50, 4524 (1994).

[36] This actually requires also the condition $U \gg|t| \ell$ to hold which is typically satisfied. No Klein factors appear in Eqs. (3) as they give rise only to an overall factor \pm 1 that can be absorbed into the tunneling matrix element [28].

[37] The result that external voltages enter as uniform constraints on chiral charge densities in the tunneling region is nontrivial. Similar relations arise for a homogeneous interacting quantum wire in the absence of backscattering. See, e.g., R. Egger and H. Grabert, Phys. Rev. B 58, 10761 (1998); I. Safi, Eur. Phys. J. B 12, 451 (1999).

[38] Note that the condition of weak tunneling used here is valid for $G_{\mathrm{J}} \ll e^{2} / 2 \pi \hbar$, yet it does not restrict the tunneling strength to the perturbative limit $L \ll L_{t}$. 